\title{
Attenuated Laser Evoked Potentials in Sarcoidosis Patients in Clinical Remission
}

\author{
Ingrid H. Korenromp ${ }^{\mathrm{a}}$, Imre P. Krabbenbos ${ }^{\mathrm{b}}$, Christiaan F. van Swol ${ }^{\mathrm{c}}$, Eric P. van Dongen ${ }^{\mathrm{b}}$, \\ Oscar J. Vogels ${ }^{\mathrm{d}}$, Jan C. Grutters ${ }^{\mathrm{a}, \mathrm{e}}$, Eduard H. Boezeman ${ }^{\mathrm{d}, \mathrm{f}}$
}

\begin{abstract}
Background: Burning sensations and pain in hands and feet occur frequently in patients with sarcoidosis. Even when features of the granulomatous inflammation have resolved and the disease is in clinical remission, pain complaints may persevere. We hypothesized that pain may be related to dysfunctioning of the A $\delta$ nociceptive processing which is responsible for transporting pain signals.
\end{abstract}

Methods: Laser Evoked Potentials were recorded in patients with sarcoidosis in clinical remission $(n=62)$ of whom $27 \%$ reported complaints of burning pain in hands and/or feet, and a group of healthy volunteers $(n=28)$. Electroencephalographic recordings following laser stimulation were evaluated in terms of N2P2-amplitude, N2P2-amplitude per ms, and N2- and P2-latencies.

Results: Sarcoidosis patients in clinical remission (irrespective of burning pain complaints) showed no significant increase in N2P2amplitude and in N2P2- amplitude per ms at stimulus intensity 2.0 Watt, while healthy controls did show a significant increase. Post hoc analysis revealed that sarcoidosis patients with burning pain complaints showed the smallest N2P2- amplitude per ms at stimulus intensity 2.0 Watt $(\mathrm{P}=0.028)$. Age did not explain the differences. No differences between groups were found on the N2- and P2-latencies.

Manuscript accepted for publication October 1, 2012

${ }^{a}$ Department of Pulmonology, Center of Interstitial Lung Diseases, St. Antonius Hospital Nieuwegein, the Netherlands

${ }^{b}$ Department of Anesthesiology, Intensive Care and Pain Medicine, St. Antonius Hospital Nieuwegein, the Netherlands

${ }^{\mathrm{c}}$ Department of Medical Physics, St. Antonius Hospital Nieuwegein, the Netherlands

${ }^{\mathrm{d}}$ Department of Clinical Neurophysiology, St. Antonius Hospital Nieuwegein, the Netherlands

${ }^{\mathrm{e}}$ Division Heart and Lungs, University Medical Center Utrecht, the Netherlands

${ }^{\mathrm{f} C o r r e s p o n d i n g ~ a u t h o r: ~ E d u a r d ~ H . ~ B o e z e m a n, ~ S t . ~ A n t o n i u s ~ H o s p i t a l, ~}$

Department of Clinical Neurophysiology, Postbus 2500, 3430 EM

Nieuwegein, the Netherlands.

Email: e.boezeman@antoniusziekenhuis.nl

doi: http://dx.doi.org/10.4021/jnr150w
Conclusions: This is the first study investigating small fiber processing in sarcoidosis patients by Laser Evoked Potentials. A $\delta$ nociceptive processing was found to be reduced in patients in which clinical signs of sarcoidosis have resolved, particularly in those reporting complaints of burning hands and/or feet. The latter data are suggestive for clinical as well as subclinical neuropathy in sarcoidosis patients after clinical remission of the disease.

Keywords: Laser evoked potentials; Sarcoidosis; Clinical remission; A $\delta$ fibers

\section{Introduction}

Pain is one of the most often reported symptom in sarcoidosis [1-3]. In particular, burning sensations and pain in hands and feet occur frequently $[4,5]$. Sarcoidosis is a systemic inflammatory granulomatous disorder of unknown cause which regularly resolves within 2 to 4 years [6]. From clinical reports $[7,8]$ and expert experience in our clinic, it is known that pain complaints may persist even when features of disease activity have resolved. In previous studies $[9,10]$ we showed that $49 \%$ of our study group that consisted of sarcoidosis patients with disease in clinical remission suffered from persisting health complaints which are predominantly characterised by pain and fatigue. These complaints had persisted up to 9 years after diagnosis. Interestingly, a high percentage of patients of this study cohort reported burning pain in hands, in feet or in both. These pain complaints point to the presence of neuropathic pain.

A possible explanation for the persistence of these neuropathic pain complaints may be found in the reports on reduction of intraepidermal nerve fiber densities (IENFD) in sarcoidosis patients with active disease. Recent studies on skin biopsies $[4,5]$ showed significantly lower intraepidermal nerve fiber densities in sarcoidosis patients compared to healthy volunteers. So, loss of small fibers in the skin has been demonstrated in sarcoidosis patients with active disease. On the basis of these data, we hypothesized that pain symptoms in patients with sarcoidosis in clinical remission 
Table 1. In- and Exclusion Criteria

Inclusion criteria:

$\begin{array}{ll}\text { Serum parameters }\left(\mathrm{sIL}-2 \mathrm{R}, \mathrm{ACE}, \mathrm{Ca}^{2+}, \mathrm{CRP}\right) & \text { normal } \\ \text { X-ray } & \text { normal or } 2 \text { years or more stable } \\ \text { Lung function } & \text { normal or } 2 \text { years or more stable, i.e.: } \\ \quad \mathrm{VC} \text { or FEV } \\ \quad \text { DLco } & <10 \% \text { change } \\ \quad & <15 \% \text { change } \\ \text { Previously involved organs } & \text { no clinical signs of disease activity }\end{array}$

Exclusion criteria:

Lofgren's syndrome

Use of corticosteroids or other immunosuppressive drugs

Use of analgesic medication

Antidepressive medication

Sleep apnea, narcolepsy or restless legs syndrome (by history)

$\mathrm{Hb}$, thrombocytes, leucocytes, $\mathrm{Na}^{2+}, \mathrm{K}^{+}$, creatinine, GGT, AF, ASAT, ALAT, LDH, gluc, protein electrophoresis, TSH, ferritine, routine urine analysis

Age

\begin{abstract}
at onset of sarcoidosis
over the last 6 months

over the last 3 months

over the last 3 months
\end{abstract}

abnormal

$>65$ years

SIL-2R: serum soluble interleukine-2 receptor, ACE: angiotensin converting enzyme, CRP: C-reactive protein, VC: vital capacity, FEV1: forced expired volume in the first second of expiration, DLco: diffusion capacity of the lung for carbon monoxide.

are possibly related to dysfunction of the small fibers as well. According to the latest European guidelines laser evoked potentials (LEP) is considered the most reliable neurophysiological tool for assessing the nociceptive pathway function in patients with neuropathic pain [11]. In short, laser stimuli selectively activate pain receptors in the skin, and generate an afferent volley in the poorly myelinated $\mathrm{A} \delta$ fibers. Upon arrival to the brain, these volleys can be recorded at the vertex as laser-evoked potentials (LEP). LEP has been proven to be a sensitive and reliable method to assess the nociceptive function of neural pathways [12-16]. Furthermore, LEP is not only an objective method to investigate pain processing but also independent on participants' motivation to actively participate in the experiment. Therefore, we tested the A $\delta$ fiber mediated nociceptive processing using LEP.

In summary, the aim of this study was to investigate whether reduced $\mathrm{A} \delta$ nociceptive processing is an explaining factor for persisting pain complaints of burning hands and/or feet in patients with sarcoidosis in clinical remission.

\section{Materials and Methods}

\section{Subjects}

One-hundred and ninety-three sarcoidosis patients (irrespective of the presence of complaints of burning pain in hands and/or feet) were invited to participate in the study. They were selected from a database of 800 sarcoidosis patients and selection criteria were: sarcoidosis had been diagnosed according to the latest ATS/ERS/WASOG statement on sarcoidosis [6]; probability to meet the in- and exclusion criteria (Table 1) on the basis of available medical records. The inclusion criteria focus on the clinical remission of sarcoidosis, while the exclusion criteria rule out other causes or disabilities that might interfere with the study outcome. Upon signing the informed consent, they were tested for inand exclusion criteria (Table 1). After screening, 62 participants were included. The patient group was split in a group which presented with complaints of burning pain sensations 

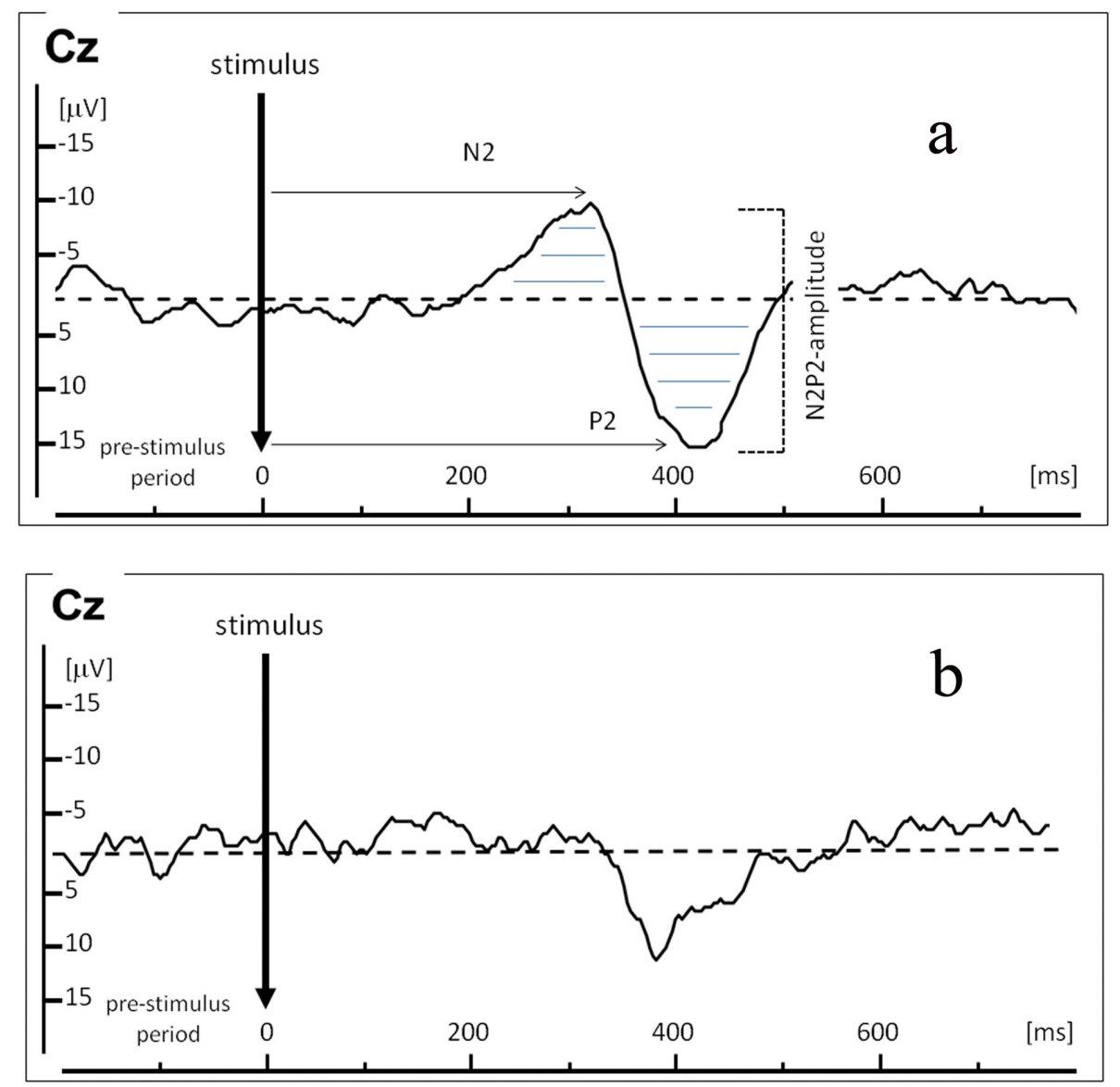

Figure 1. Example of a Laser Evoked Potential (LEP) recordings in one representative healthy control (a), and below an example of an attenuated LEP in one representative sarcoidosis patient in clinical remission with complaints of burning pain (b). X-axis represents time in ms; Y-axis represents amplitude in $\mu \mathrm{V}$. LEP was recorded at electrode $\mathrm{Cz}$. Arrows indicate N2-latency and P2-latency in ms; horizontal lines indicate N2P2-amplitude $(\mu \mathrm{V})$ over time $(\mathrm{ms})$.

in hands and/or feet, and a group in which these complaints were absent.

In addition, 28 healthy volunteers, recruited from hospital personnel, had been tested with Laser Evoked Potentials. These data had already been sampled 2 years before the start of the present study. Finally, we like to emphasize that none of the participants received analgesic treatment at the time of the study.

The Medical Research Ethics Committee of the St. Antonius Hospital Nieuwegein, the Netherlands approved the study protocol, MEC ID: R-06.38A, GOV ID: NL14786.100.06.

\section{Laser and stimulation protocol}

Cutaneous heat stimuli were delivered by a $980-\mathrm{nm}$ diode laser (Biolitec, Ceram Optec, Bonn, Germany) to the blackened dorsum of the right foot. Blackening of the skin was performed to rule out bias by differences in reflectance of energy by skin pigmentation $[17,18]$. Laser intensity varied between 1.0 and 2.0 Watt $\left(10-20 \mathrm{~mJ} / \mathrm{mm}^{2}\right)$, duration of the stimuli was set at $50 \mathrm{~ms}$, with a laser beam spot size of 5 $\mathrm{mm}^{2}$. The stimulus intensity was adjusted to evoke a clear pinprick sensation. Interstimulus duration randomly varied between 5 and 10 seconds to assure reorientation of attention to an unexpected stimulus.

The stimulation protocol comprised 6 runs of each 10 stimuli, in the following sequence: 1.0 Watt, 2.0 Watt, 1.5 Watt, 1.5 Watt, 2.0 Watt, and 1.0 Watt. In order to minimize skin irradiation and nociceptor habituation the laser target was slightly displaced after each stimulation. Before the stimulation protocol was started, a single reference laser stimulus of 1.0 Watt was administered to verify whether this intensity was supra-threshold in all patients. Participants and experimenters wore protective goggles throughout the experiment.

\section{Recording and processing}

Electroencephalography (EEG) recordings (Fig. 1) were 
Table 2. Characteristics of Healthy Controls, Sarcoidosis Patients in Clinical Remission Split in a Group With Complaints of Burning Pain and a Group With no Complaints of Burning Pain

\begin{tabular}{|c|c|c|c|c|}
\hline & \multirow{2}{*}{$\begin{array}{l}\text { Healthy } \\
\text { controls }\end{array}$} & \multicolumn{2}{|c|}{ Sarcoidosis in clinical remission } & \multirow[t]{2}{*}{$\mathbf{P}$} \\
\hline & & $\begin{array}{l}\text { with burning } \\
\text { pain }\end{array}$ & $\begin{array}{l}\text { no burning } \\
\text { pain }\end{array}$ & \\
\hline$n$ & 28 & 17 & 45 & \\
\hline Report on pain in: & 0 & & 0 & \\
\hline hands & & 7 (2 males) & & \\
\hline feet & & 5 (2 males) & & \\
\hline hands and feet & & 5 (2 males) & & \\
\hline Gender & & & & 0.341 \\
\hline male & 13 & 6 & 19 & \\
\hline female & 15 & 11 & 26 & \\
\hline Age in years $($ mean $\pm s d)$ & $34 \pm 8$ & $46 \pm 9$ & $47 \pm 7$ & $<0.001 *$ \\
\hline $\begin{array}{l}\text { Time since diagnosis in years } \\
(\text { median }(\mathrm{IQR}))\end{array}$ & & $7(5-10)$ & $11(6-18)$ & 0.002 \\
\hline $\begin{array}{l}\text { Total number of affected organs (mec } \\
(\mathrm{IQR}) \text { ) }\end{array}$ & & $2(1-3)$ & $2(1-2)$ & 0.787 \\
\hline \multicolumn{5}{|l|}{ Former use of corticosteroids } \\
\hline yes & & 6 & 17 & 0.769 \\
\hline months of use (median (IQR)) & & $16(0.5-30)$ & $16(11-22)$ & 0.254 \\
\hline \multicolumn{5}{|l|}{ Sensory sum score \# } \\
\hline total & & $54.23 \pm 3.4$ & $55.33 \pm 1.8$ & 0.184 \\
\hline \multicolumn{5}{|l|}{ touch and pinprick sensation } \\
\hline arm & & $28.00 \pm 0$ & $27.95 \pm 0.3$ & 0.539 \\
\hline leg & & $26.00 \pm 3.9$ & $26.98 \pm 2.5$ & 0.243 \\
\hline vibration sense & & $18.94 \pm 2.1$ & $19.64 \pm 1.1$ & 0.082 \\
\hline joint position sensation & & $7.17 \pm 1.4$ & $7.73 \pm 0.8$ & 0.073 \\
\hline Atrophy Extensor Digitorum Brevis & & & & 0.467 \\
\hline absent & & 16 & 44 & \\
\hline present & & 1 & 1 & \\
\hline Achilles Reflex & & & & 0.527 \\
\hline normal & & 11 & 35 & \\
\hline reduced & & 3 & 6 & \\
\hline absent & & 3 & 4 & \\
\hline
\end{tabular}

Significant differences in bold. * Significant difference between healthy controls and patients with burning pain; and between healthy controls and patients without burning pain; \# Sensory sum score was measured as follows: touch and pinprick sensation: 4: normal, 3: abnormal distal to wrist/ankle, 2: abnormal distal to half forearm/leg, 1: abnormal distal to knee/elbow, 0: abnormal distal to groin/axilla. Vibration sense: $128 \mathrm{~Hz}$ tuning fork perception on: 4: middle finger/hallux, 3: ulnar styloid/medial malleolus, 2: elbow/knee, 1: clavicle/iliac crest, 0: absent. Joint position sensation of middle finger/ hallux: 2: normal, 1: diminished, 0: absent. 


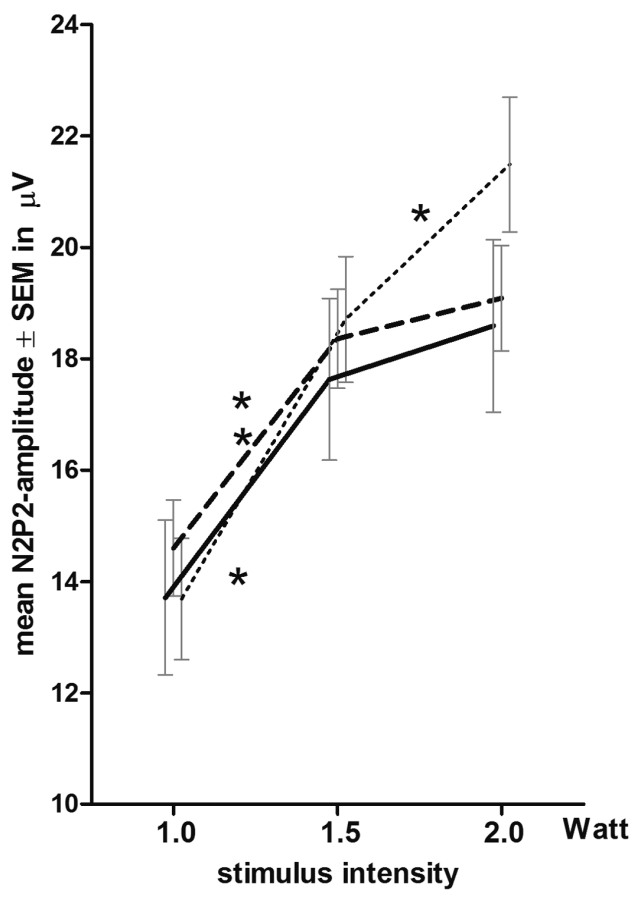

$$
\begin{aligned}
& \text { - complaints of burning pain } \\
& \text {-- no burning pain } \\
& \text { *-. healthy controls } \\
& \text { * significant increase between } 2 \text { stimulus intensities } \\
& \text { no significant differences between } 3 \text { groups at } 2.0 \text { Watt }
\end{aligned}
$$

Figure 2. Mean \pm SEM N2P2-amplitude on laser intensity 1.0, 1.5 and 2.0 Watt

made to measure brain activity following laser stimulation. Silver disk electrodes were positioned on the skull according to the International 10-20 system. Cz, C3, C4, and $\mathrm{Pz}$ electrodes from the vertex were linked to both ear lobes (A1, A2). Impedance was kept below $5 \mathrm{kOhm}$. Electrooculography was recorded for eye movement artifact filtering. Online single sweep EEG monitoring was performed. Recordings showing blinks, eye movements, or any other artifacts were manually deleted followed by offline response averaging and analysis. EEG signals were sampled at $1000 \mathrm{~Hz}$ after bandpass filtering $(0.5-30 \mathrm{~Hz})$ by Viasys Healthcare Inc. (Viasys Healthcare Inc., Houten, the Netherlands). EEG epochs were recorded between $200 \mathrm{~ms}$ before until $800 \mathrm{~ms}$ after the onset of each stimulus.

\section{Statistical analysis}

The extent of the laser evoked potentials was presented as N2P2-amplitude (in $\mu \mathrm{V}$ ) and N2- and P2-latency (in ms). In addition, the N2P2-amplitude over the period of time during which this volley was present (N2P2-amplitude per ms) was calculated (in $\mu \mathrm{V} . \mathrm{ms}$ ), also known as the Area Under the Curve (AUC). Incorporating this time effect may be important because it might reflect the evoked potentials more robustly than just the absolute maximum of the N2P2-amplitude. Next, the mean N2P2-amplitude, mean N2- and P2-latency and mean N2P2-amplitude per ms were computed for every participant per stimulus intensity by offline averaging of corresponding stimulation blocks.

As LEP recordings were repeated measurements, the analysis was carried out with the Linear Mixed Models repeated measurement approach in SPSS version 16.0. Data on demographic variables and questionnaires scores were analysed with one way ANOVA and Chi-square tests. In all analyses, the significance level was set at $\mathrm{P}<0.05$.

\section{Results}

\section{Study population}

Sixty-two patients with histological proven sarcoidosis were 


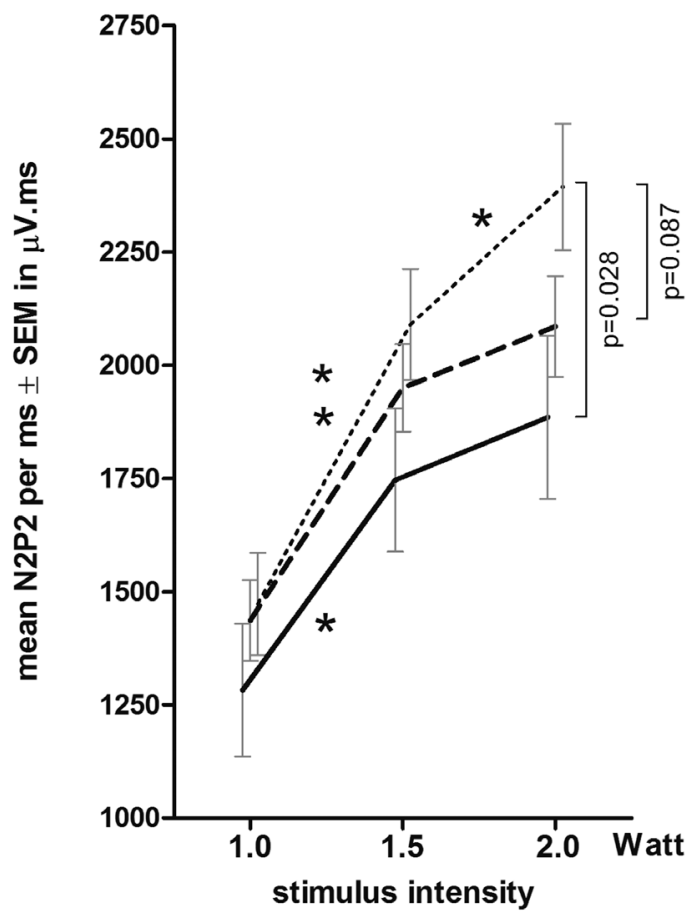

$$
\begin{aligned}
& \text { - complaints of burning pain } \\
& \text {-- no burning pain } \\
& \text {-.- healthy controls } \\
& \text { * } \quad \text { significant increase between } 2 \text { stimulus intensities }
\end{aligned}
$$

Figure 3. Mean \pm SEM N2P2-amplitude per ms on laser intensity 1.0, 1.5 and 2.0 Watt.

included. In all patients no disease activity could be detected: serum parameters (ACE, serum sIL2-R, CRP, calcium) were normal; lung function was within normal reference scores. Physical examination at time of study revealed no clinical signs of disease activity in any organs. X-ray revealed no abnormalities in all patients, except for one (fatigued) patient who presented with slight bihilair lymphadenopathy. Median time since diagnosis was 9.5 years, with interquartile range of 6 to 17 years. Seventeen out of 62 patients $(27 \%)$ reported complaints of burning pain in either hands, or feet, or both.

Characteristics of the study group are summarized in Table 2. No differences were found between groups with respect to gender, total number of affected organs, history of corticosteroid use and neurological examination. Time since diagnosis was significantly shorter in the group with burning pain complaints than in the group with no burning pain complaints. The group of healthy controls was significantly younger ( $34 \pm 8 \mathrm{yrs})$ than the patient group $(46.5 \pm 8 \mathrm{yrs}, \mathrm{P}<$ 0.001 ). Therefore, we tested whether the increase in N2P2amplitude, N2P2-amplitude per ms, and N2- and P2 latencies could be explained by differences in age by adding 'age' as a fixed factor to the statistical model.

\section{Laser evoked potentials}

\section{N2P2-amplitude}

Overall analysis revealed that the increase of stimulus intensity elicited a significant increase in N2P2-amplitude (F (2, $93)=47.074, \mathrm{P}<0.0001$ ), irrespective of group membership $(\mathrm{F}(2,85)=0.476, \mathrm{P}=0.623)$. The interaction between stimulus intensity and group membership did not significantly differ $(F(4,93)=1.567, P=0.190)$. Next, post hoc analyses were performed.

Post hoc analysis within each group revealed that no significant increase in N2P2-amplitude was observed on stimulus intensity 2.0 Watt in both the group with burning pain and the group without burning pain $(\mathrm{P}=0.402$ and $\mathrm{P}=0.501)$, while a significant increase was present in the healthy controls $(\mathrm{P}=0.014)$. Figure 2 shows the significant increases in N2P2-amplitudes between two stimulus-intensities (indicated with an asterisk $(*))$.

Furthermore, post hoc pairwise comparison of groups showed that, although trends are suggested in Figure 2, no statistical significance was reached between groups on stim- 
ulus intensity 2.0 Watt (healthy controls versus burning pain group $(\mathrm{P}=0.116)$ and versus group with no burning pain ( $\mathrm{P}$ $=0.108)$.

Age was no significant contributor to the increase of N2P2-amplitude $(\mathrm{F}(50,34)=0.62, \mathrm{P}=0.938)$.

\section{N2P2-amplitude per ms}

A similar pattern was assessed when taking the time effect into account: the increase of stimulus intensity induced a significant increase in N2P2-amplitude per $\mathrm{ms}(\mathrm{F}(2,91)=63.394$, $\mathrm{P}<0.0001)$. Group membership as well as the interaction between stimulus intensity and group membership turned out to be non-significant $(\mathrm{F}(2,88)=2.086, \mathrm{P}=0.130$ respectively $\mathrm{F}$ $(4,91)=0.979, \mathrm{P}=0.423)$. Next, post hoc analyses were performed. Post hoc analysis within each group revealed that no significant increase in N2P2-amplitude per ms was observed on stimulus intensity 2.0 Watt in both the group with burning pain and the group with no burning pain $(\mathrm{P}=0.414$ and $\mathrm{P}=$ $0.195)$, while a significant increase was present in the healthy controls ( $\mathrm{P}=0.024)$ (Fig. 3).

In addition, post hoc pairwise comparison of groups showed that compared with the healthy controls the N2P2amplitude per ms on stimulus intensity 2.0 Watt was significantly smaller in the group with burning pain $(\mathrm{P}=0.028)$ and decreased in the group with no burning pain $(\mathrm{P}=0.087)$ (Fig. $3)$. Age did not significantly contribute to the increase of the N2P2-amplitude per ms $(\mathrm{F}(50,34)=0.56, \mathrm{P}=0.967)$.

\section{N2- and P2-latencies}

Increasing stimulus intensity did not elicit a raise in N2-latency or in $\mathrm{P} 2$-latency $(\mathrm{P}=0.686$ and $\mathrm{P}=0.143)$. No group effect and no interaction effect between stimulus intensity and group membership were observed in N2-latency as well as in P2-latency. Age did not contribute to both latencies ( $\mathrm{F}(50,34)$ $=0.79, \mathrm{P}=0.773$, respectively $\mathrm{F}(50,34)=1.05, \mathrm{P}=0.438)$.

\section{Discussion}

This is the first study which investigates pain processing via A $\delta$-fibers in sarcoidosis patients in clinical remission of the disease by Laser Evoked Potentials (LEP). We are the first to show that, compared to healthy controls, $\mathrm{A} \delta$ nociceptive processing is significantly reduced in sarcoidosis patients in clinical remission, particularly in the patients that report complaints of burning hands and/or feet.

No significant increases in N2P2-amplitude and timecorrected N2P2-amplitude (amplitude per ms) at stimulus intensity 2.0 Watt were observed in sarcoidosis patients in clinical remission (irrespective of burning pain complaints), whereas increases in these parameters did show significance in healthy controls. Moreover, laser evoked N2P2-ampli- tude and N2P2- amplitude per ms on stimulus intensity 2.0 Watt were the smallest in the clinical remission group with complaints of burning pain in hand and/or feet compared to healthy controls.

Reduced N2P2-amplitude in response to LEP points to a dysfunction of the nociceptive was processing of A $\delta$ fibers. This dysfunction may be present anywhere in the sensory pathway from the skin to the cortex. Latency data (N2- and P2-latencies) did not differ between the groups, suggesting a normal conduction time for ascending volleys reaching the cortex following laser stimulation of the skin. Therefore, a demyelinating lesion in those pathways is not very likely. Considering the fact that in sarcoidosis a loss of intraepidermal nerve fibers has been demonstrated using skin biopsy [4, 5], the reduced $\mathrm{N} 2 \mathrm{P} 2$-amplitude in the group with burning pain complaints may well be attributed to this phenomenon. An additional positive argument for this assumption is the recent report of a strong relationship between attenuated LEP and intraepidermal nerve fiber density [19]. We therefore propose that the attenuated LEP in sarcoidosis patients in clinical remission may be the result of axonal loss of the thinly myelinated A $\delta$ fibers in the skin.

Interestingly, this study showed as well that compared to healthy controls the reduced N2P2-amplitude and N2P2amplitude per ms were present in both patients with complaints of burning hands and/or feet as well as in those without these complaints. So, irrespective of occurrence of these neuropathic pain complaints, one may put forward that all patients who are in clinical remission of sarcoidosis may (to some extent) face long term (micro-) neuronal damage which might only become apparent by measurements such as LEP. So far, LEP has never been studied in sarcoidosis patients in clinical remission nor in sarcoidosis patients without burning pain complaints. Our novel and unique observation of reduced $A \delta$ nociceptive processing in this asymptomatic remission group is issue for discussion. A possible explanation might be sought in the preceding disease. Sarcoidosis may have triggered alterations in the nociceptive processing to different extents in both patient groups. According to the literature, immune-mediated diseases $[20,21]$ of which sarcoidosis is one, may induce damage to small fibers [22]. Sarcoidosis is a systemic inflammatory disorder which is immunologically characterized by elevated levels of interleukine-2 (IL-2), interferon-gamma (IFN- $\gamma$ ), and subsequently macrophage tumor necrosis factor-alpha (TNF- $\alpha)$. It is likely that the cause of the neuropathy may be attributed to this inflammation. In particular TNF- $\alpha$ is known for its role in inciting damage to nerves and surrounding tissues [23]. Moreover, high levels of TNF- $\alpha$ may result in activation and morphological changes of microglial cells in the spinal cord. The latter process may lead to central sensitization which will provoke pain sensations a long time after the resolution of the initial inflammation [24]. Recovery from central sensi- 
tization may take years. In this light we like to remark that the median time since diagnosis was significantly longer in the group without complaints of burning pain (11 years) than in the group with burning pain complaints (7 years). It would be most interesting to follow the latter group and determine whether their complaints will improve over time. In addition, more research is needed to definitely prove the mechanism of perpetuating deteriorated nociceptive processing when signs of sarcoidosis have long resolved.

A limitation of the study is that the data of the healthy control group was sampled 2 years before the start of the present study. As a result, this group did not match the age of the patient group. The mean age of the healthy control group was significantly lower than the mean age of both patient groups. Although lower intraepidermal fiber nerve densities are found in older age $[4,25]$, age did not show a significant effect on the increase of N2P2-amplitude, N2- and P2latency, and N2P2- amplitude per ms in our study cohort. Therefore, we conclude that the reduced N2P2-amplitude and N2P2- amplitude per ms which we found in the group with burning pain complaints can not be explained by differences in age.

Moreover, the data have not only been presented as N2P2-amplitude and N2- and P2-latency, but also as N2P2amplitude per ms. We do realize that the latter presentation is innovative. N2P2- amplitude per ms is not the generally accepted standard in the field of LEP, while it is in other research areas such as endocrinology and neurosciences. Nevertheless, calculating the N2P2-amplitude over the period of time during which this volley was present may supply additional information about the $\mathrm{A} \delta$ nociceptive processing. In the present study for instance, statistical significance in $\mathrm{N} 2 \mathrm{P} 2$-amplitude was not reached in the post hoc pairwise comparison of groups, while differences in N2P2- amplitude per ms between the group of healthy controls and patient group with burning pain was statistically significant. This is an interesting observation. In our opinion, it might be attributed to the two-dimensional aspect of the N2P2- amplitude per ms. Besides the absolute maximum of the N2P2amplitude, the N2P2- amplitude per ms also incorporates a time effect. Presenting our data as N2P2- amplitude per ms has not only revealed that in the patient group the peaks of the N2 and P2 were lower (reduced N2P2-amplitude), but has also shown that the shape of curve of these volleys were significantly decreased, particularly in those patients with complaints of burning pain in hands and/or feet. From this point of view the N2P2- amplitude per ms might be considered as a more sensitive reflection of the laser evoked potential. Nevertheless, this remarkable observation is worth additional research.

\section{Conclusions}

In conclusion, this is the first study that investigates pain processing via $A \delta$ fibers in sarcoidosis patients in clinical remission of the disease by Laser Evoked Potentials. We are the first to show that, compared to healthy controls, A $\delta$ nociceptive processing is significantly reduced in sarcoidosis patients in clinical remission, in particular in those patients with complaints of burning pain in hands and/or feet.

\section{Acknowledgement}

The authors wish to thank Pieter Zanen for his excellent statistical advice.

\section{Clinical Implications}

These study results are suggestive for clinical as well as subclinical neuropathy in sarcoidosis patients who are in clinical remission. This (sub-) clinical neuropathy might have implications for evaluation and rehabilitation approaches used by physicians.

\section{Disclosure}

Authors have no conflicts of interest to declare.

\section{References}

1. Hoitsma E, De Vries J, van Santen-Hoeufft M, Faber CG, Drent M. Impact of pain in a Dutch sarcoidosis patient population. Sarcoidosis Vasc Diffuse Lung Dis. 2003;20(1):33-39.

2. Michielsen HJ, Peros-Golubicic T, Drent M, De Vries J. Relationship between symptoms and quality of life in a sarcoidosis population. Respiration. 2007;74(4):401405 .

3. Wirnsberger RM, de Vries J, Breteler MH, van Heck GL, Wouters EF, Drent M. Evaluation of quality of life in sarcoidosis patients. Respir Med. 1998;92(5):750-756.

4. Bakkers M, Merkies IS, Lauria G, Devigili G, Penza P, Lombardi R, Hermans MC, et al. Intraepidermal nerve fiber density and its application in sarcoidosis. Neurology. 2009;73(14):1142-1148.

5. Bakkers M, Faber CG, Drent M, Hermans MC, van Nes SI, Lauria G, De Baets M, et al. Pain and autonomic dysfunction in patients with sarcoidosis and small fibre neuropathy. J Neurol. 2010;257(12):2086-2090.

6. Costabel U, Hunninghake GW. ATS/ERS/WASOG statement on sarcoidosis. Sarcoidosis Statement Committee. American Thoracic Society. European Respiratory Society. World Association for Sarcoidosis and Other Granulomatous Disorders. Eur Respir J. 1999;14(4):735-737. 
7. James DG. Complications of sarcoidosis. Chronic fatigue syndrome. Sarcoidosis. 1993;10(1):1-3.

8. Drent M. Sarcoidosis and fatigue. Modern Medicine 2003: 830-835.

9. Korenromp IH, Heijnen CJ, Vogels OJ, van den Bosch JM, Grutters JC. Characterization of chronic fatigue in patients with sarcoidosis in clinical remission. Chest. 2011;140(2):441-447.

10. Korenromp IH, Grutters JC, van den Bosch JM, Zanen P, Kavelaars A, Heijnen CJ. Reduced Th2 cytokine production by sarcoidosis patients in clinical remission with chronic fatigue. Brain Behav Immun. 2011;25(7):14981502.

11. Cruccu G, Sommer C, Anand P, Attal N, Baron R, Garcia-Larrea L, Haanpaa M, et al. EFNS guidelines on neuropathic pain assessment: revised 2009. Eur J Neurol. 2010;17(8):1010-1018.

12. Treede RD, Lorenz J, Baumgartner U. Clinical usefulness of laser-evoked potentials. Neurophysiol Clin. 2003;33(6):303-314

13. Truini A, Panuccio G, Galeotti F, Maluccio MR, Sartucci F, Avoli M, Cruccu G. Laser-evoked potentials as a tool for assessing the efficacy of antinociceptive drugs. Eur J Pain. 2010;14(2):222-225.

14. Truini A, Galeotti F, Romaniello A, Virtuoso M, Iannetti GD, Cruccu G. Laser-evoked potentials: normative values. Clin Neurophysiol. 2005;116(4):821-826.

15. Garcia-Larrea L, Convers P, Magnin M, Andre-Obadia N, Peyron R, Laurent B, Mauguiere F. Laser-evoked potential abnormalities in central pain patients: the influence of spontaneous and provoked pain. Brain. 2002;125(Pt 12):2766-2781.

16. Krabbenbos IP, Brandsma D, van Swol CF, Boezeman EH, Tromp SC, Nijhuis HJ, van Dongen EP. Inhibition of cortical laser-evoked potentials by transcutaneous electrical nerve stimulation. Neuromodulation. 2009;12(2):141-145.
17. Plaghki L, Mouraux A. How do we selectively activate skin nociceptors with a high power infrared laser? Physiology and biophysics of laser stimulation. Neurophysiol Clin. 2003;33(6):269-277.

18. Arendt-Nielsen L, Bjerring P. Sensory and pain threshold characteristics to laser stimuli. J Neurol Neurosurg Psychiatry. 1988;51(1):35-42.

19. Casanova-Molla J, Grau-Junyent JM, Morales M, VallsSole J. On the relationship between nociceptive evoked potentials and intraepidermal nerve fiber density in painful sensory polyneuropathies. Pain. 2011;152(2):410418.

20. Devigili G, Tugnoli V, Penza P, Camozzi F, Lombardi R, Melli G, Broglio L, et al. The diagnostic criteria for small fibre neuropathy: from symptoms to neuropathology. Brain. 2008;131(Pt 7):1912-1925.

21. Uceyler N, Devigili G, Toyka KV, Sommer C. Skin biopsy as an additional diagnostic tool in non-systemic vasculitic neuropathy. Acta Neuropathol. 2010;120(1):109116.

22. Khan S, Zhou L. Characterization of non-length-dependent small-fiber sensory neuropathy. Muscle Nerve. 2012;45(1):86-91.

23. Leung L, Cahill CM. TNF-alpha and neuropathic pain-a review. J Neuroinflammation. 2010;7:27.

24. Andrade P, Visser-Vandewalle V, Hoffmann C, Steinbusch HW, Daemen MA, Hoogland G. Role of TNFalpha during central sensitization in preclinical studies. Neurol Sci. 2011;32(5):757-771.

25. Lauria G, Hsieh ST, Johansson O, Kennedy WR, Leger JM, Mellgren SI, Nolano M, et al. European Federation of Neurological Societies/Peripheral Nerve Society Guideline on the use of skin biopsy in the diagnosis of small fiber neuropathy. Report of a joint task force of the European Federation of Neurological Societies and the Peripheral Nerve Society. Eur J Neurol. 2010;17(7):903912, e944-909. 